\title{
Video Article \\ Generation of Human 3D Lung Tissue Cultures (3D-LTCs) for Disease Modeling
}

\author{
Michael Gerckens ${ }^{1,2,3}$, Hani N. Alsafadi ${ }^{4,5,6}$, Darcy E. Wagner ${ }^{4,5,6}$, Michael Lindner ${ }^{2,7}$, Gerald Burgstaller ${ }^{*^{1,2,3}}$, Melanie Königshoff ${ }^{\star 1,2,3,8}$ \\ ${ }^{1}$ Comprehensive Pneumology Center, Ludwig-Maximilians-Universität and Helmholtz Zentrum Munich \\ ${ }^{2}$ German Center of Lung Research (DZL) \\ ${ }^{3}$ Translational Lung Research and CPC-M bioArchive, Helmholtz Zentrum München, Comprehensive Pneumology Center Munich DZL/CPC-M \\ ${ }^{4}$ Department of Experimental Medical Science, Lung Bioengineering and Regeneration, Lund University \\ ${ }^{5}$ Wallenberg Center for Molecular Medicine, Lund University \\ ${ }^{6}$ Stem Cell Centre, Lund University \\ ${ }^{7}$ Asklepios Fachkliniken Munich-Gauting \\ ${ }^{8}$ Department of Medicine, Division of Pulmonary Sciences and Critical Care Medicine, University of Colorado \\ *These authors contributed equally
}

Correspondence to: Gerald Burgstaller at gerald.burgstaller@helmholtz-muenchen.de, Melanie Königshoff at melanie.koenigshoff@ucdenver.edu

URL: https://www.jove.com/video/58437

DOI: doi: $10.3791 / 58437$

Keywords: Medicine, Issue 144, 3D tissue culture, ex-vivo tissue culture, precision-cut lung slices, PCLS, 3D-LTCs, lung disease, disease animal models, human ex-vivo models, lung fibrosis

Date Published: 2/12/2019

Citation: Gerckens, M., Alsafadi, H.N., Wagner, D.E., Lindner, M., Burgstaller, G., Königshoff, M. Generation of Human 3D Lung Tissue Cultures (3D-LTCs) for Disease Modeling. J. Vis. Exp. (144), e58437, doi:10.3791/58437 (2019).

\section{Abstract}

Translation of novel discoveries to human disease is limited by the availability of human tissue-based models of disease. Precision-cut lung slices (PCLS) used as 3D lung tissue cultures (3D-LTCs) represent an elegant and biologically highly relevant 3D cell culture model, which highly resemble in situ tissue due to their complexity, biomechanics and molecular composition. Tissue slicing is widely applied in various animal models. 3D-LTCs derived from human PCLS can be used to analyze responses to novel drugs, which might further help to better understand the mechanisms and functional effects of drugs in human tissue. The preparation of PCLS from surgically resected lung tissue samples of patients, who experienced lung lobectomy, increases the accessibility of diseased and peritumoral tissue. Here, we describe a detailed protocol for the generation of human PCLS from surgically resected soft-elastic patient lung tissue. Agarose was introduced into the bronchoalveolar space of the resectates, thus preserving lung structure and increasing the tissue's stiffness, which is crucial for subsequent slicing. $500 \mu \mathrm{m}$ thick slices were prepared from the tissue block with a vibratome. Biopsy punches taken from PCLS ensure comparable tissue sample sizes and further increase the amount of tissue samples. The generated lung tissue cultures can be applied in a variety of studies in human lung biology, including the pathophysiology and mechanisms of different diseases, such as fibrotic processes at its best at (sub-)cellular levels. The highest benefit of the 3D-LTC ex vivo model is its close representation of the in situ human lung in respect of 3D tissue architecture, cell type diversity and lung anatomy as well as the potential for assessment of tissue from individual patients, which is relevant to further develop novel strategies for precision medicine.

\section{Video Link}

The video component of this article can be found at https://www.jove.com/video/58437/

\section{Introduction}

Chronic and acute lung diseases are a major cause of morbidity and mortality worldwide ${ }^{1}$. For patients with chronic lung diseases like obstructive pulmonary disease (COPD) ${ }^{2}$, severe asthma ${ }^{3}$, lung cancer ${ }^{4}$ and diffuse parenchymal lung diseases ${ }^{5}$, curative therapies are currently not available. Although studies in animal models for lung diseases have deepened the understanding of disease pathomechanisms ${ }^{6}$ and have led to the identification of potential novel therapeutic targets ${ }^{7,8,9}$, these models exhibit relevant biological and physiological differences when compared to humans ${ }^{10}$. To overcome these discrepancies between murine and human biology as well as anatomy, human ex vivo 3D lung tissue culture (3D-LTC) systems are used in various areas of biomedical research. These 3D-LTC culture systems are based on precision-cut lung slices (PCLS). The generation of PCLS ex vivo allows analysis of a third spatial dimensionality, which allows for the investigation of the spatial and functional relationships of cells in entire alveoli and airways ${ }^{11}$, as well as the interstitium, vasculature and mesothelium. Notably, PCLS ex vivo models are multicellular, meaning that they contain most functional cells of in situ lungs, thus closely representing the cells' native biological environment and thus overcoming the limited cell-cell and cell-matrix interaction in most 2D cell culture approaches. Up to now, ex vivo murine PCLS were used to model pulmonary diseases, like COPD ${ }^{12}$, lung fibrosis ${ }^{13}$, lung cancer $^{14}$, viral infection ${ }^{15,16}$, bronchopulmonary dysplasia ${ }^{17}$, and asthma ${ }^{18}$. However, a considerable proportion of novel drug therapies in human lung diseases that were investigated in clinical trials do not 
translate to the clinic due to their lack of efficacy or safety, assumingly due to yet considerable differences between human and murine biology and disease $e^{19,20,21}$.

Over several years, human PCLS have been largely used to assess lung toxicity of chemicals and drugs. Only recently, human lung tissue has been used from patients with COPD $^{22,23}$, asthma ${ }^{24}$, and lung fibrosis ${ }^{25}$, to pursue pathophysiological and pharmacological studies. By using resected patient organ material and generating PCLS thereof, one can recapitulate major disease hallmarks in a complex 3D tissue environment ${ }^{22}$ representing and maintaining most of the native cellular diversity of the organ. Moreover, diseased tissue applied in a variety of experimental setups was shown to mimic disease-like changes in liver, intestine and kidney ${ }^{26,27,28,29}$.

However, processing of lung tissue remains challenging for several reasons. Unlike solid tissue, native lung parenchyma tends to collapse without ventilation and exhibits lower tissue stiffness. These properties impede the slicing of the tissue. Thus, filling of airways and the alveolar space with low-melting point agarose preserves the native lung structure and provides the stiffness needed for precision-cut slicing of murine and human lungs ${ }^{30}$. Human lung resectates donated for research purposes are by their nature anatomically, genetically and physiologically highly diverse, thus often presenting a high inter-patient variability when performing experiments ${ }^{25}$. In contrast to the whole lobe or whole lung explants, lung samples resected by means of thoracic surgery do not necessarily follow the anatomical segments and, therefore, require special preparation. In this article, we provide a detailed and optimized protocol for the generation of human PCLS from resected lung tissue and their subsequent cultivation and experimental use to model lung disease.

\section{Protocol}

The use of human tissue was approved by the ethics committee of the Ludwig-Maximillian University [Munich, Germany (project number 455-12)]. Tumor-free human lung resections were provided by the Asklepios Biobank for Lung Diseases (Gauting, Germany, project number 333-10).

NOTE: All procedures of human PCLS production (Figure 1) are done under a sterile laminar flow hood.

\section{Preparation of Instruments and Materials}

1. Prepare all materials for the inflation of lung tissue with agarose as described below.

1. Prepare the cultivation medium: Dulbecco's Modified Eagle Medium (DMEM) F-12 supplemented with L-Glutamine, HEPES, 10,000 IE Penicillin, 10,000 IE Streptomycin and $0.1 \%(v / v)$ fetal bovine serum.

NOTE: Medium is used at $37^{\circ} \mathrm{C}$.

2. Prepare a sterile metal tray covered with tissue paper. Place a sterile $15 \mathrm{~cm}$ cell culture dish on the tray.

3. Fill the cell culture dish with the $15 \mathrm{~mL}$ of cultivation medium.

4. Prepare a $3 \%(\mathrm{w} / \mathrm{v})$ agarose solution by dissolving the appropriate amount of low-melting point agarose in a minimum of $30 \mathrm{~mL}$ of cultivation medium.

5. Heat the solution in a microwave until boiling. Cool the agarose solution to $42{ }^{\circ} \mathrm{C}$ in a water bath. Keep the liquid agarose solution stored in the water bath.

6. Prepare several $50 \mathrm{~mL}$ conical tubes filled with the liquid agarose.

\section{Resected Lung Tissue}

1. Store fresh tumor free lung tissue of lobectomy resectates immediately after resection in DMEM F-12 medium at $4{ }^{\circ} \mathrm{C}$ until step 3 .

2. Do not exceed cold ischemia time of $4-8 \mathrm{~h}$ prior to processing.

\section{Inspection and Selection of Resected Tissue Prior to Agarose Filling}

1. Lift the tissue from the medium with tweezers. In order to avoid any damage to the tissue, especially to the pleura, handle the tissue with tweezers at the airways only.

2. Score the tissue quality by criteria of the Lung Agarose Filling Score defined in Table 1.

3. Proceed to step 4 if the tissue's quality is scored above or equal to 72 . If the tissue's quality is scored below 60 , do not continue further with agarose filling.

NOTE: If the tissue score is between 60 and 68, the agarose-filling and tissue slicing still might produce reasonable results, and a final decision for prolongation of the experiment has to be made case to case. However, lung tissue that did not meet the above-mentioned requirements, mostly fails in agarose filling.

\section{Lung Tissue Inflation by Agarose Filling}

1. Lift the tissue from the storage medium and drain excess media from the tissue. Transfer the lung tissue into the $15 \mathrm{~cm}$ culture dish prepared in 1.1.2.

2. Fill a $30 \mathrm{~mL}$ syringe with the low-melting point agarose from 1.1.3.

3. Prepare a peripheral venous catheter by removing the obturator and attach it to a $30 \mathrm{~mL}$ syringe

4. Identify a bronchus $(0.5-3 \mathrm{~mm}$ in diameter) in the tissue that is ventilating an intact section of the tissue (see Figure 2).

5. Insert the cannula into the selected bronchus $(0.5-3 \mathrm{~mm}$ diameter).

6. Gently push the cannula gently forward as far as possible. 
7. Seal the bronchus around the cannula by compressing the bronchial wall around the cannula with forceps, ideally clamping any adjacent pulmonary artery at the same time.

8. Occlude other additional airways with a surgical clamp to prevent agarose leaking through these airways.

9. Lift the tissue with the forceps from the culture dish.

10. Manually pour the agarose with the syringe no faster than $0.3 \mathrm{~mL} / \mathrm{s}$. The speed of agarose filling might vary between approximately 0.05 and $0.3 \mathrm{~mL} / \mathrm{s}$ due to the heterogeneous resistance of airways and/or atelectasis.

11. If high resistance while filling or agarose leaking from the tissue is observed, retry the whole procedure with a different bronchus from step 4.4. Perform troubleshooting as described below.

NOTE: The degree of agarose filling is highly dependent on the position of the catheter in the tissue and deep penetration of the catheter results in agarose filling of small cone like regions $\left({ }^{*}\right)$ of the lung tissue (Figure $\mathbf{2 C}$ ).

1. In case of high resistance, try positioning of the catheter leads to proper filling of most regions of the tissue (\#) (Figure 2D).

2. As plugs of early solidified agarose in the proximal bronchi or other airway obstructions (arrow) can lead to an incomplete filling of the tissue (Figure 2E), do not force agarose filling as this might lead to defects in the filled area, but not in a filling of the obstructed tissue parts.

3. If the respiratory tree derived from the cannulated bronchus is damaged during resection and the agarose filling results in a constant leaking of the liquid agarose (arrow in Figure 2F), insert the catheter into a more peripheral part of the airway system to fill at least a minor part of the tissue $\left({ }^{*}\right)$ (Figure $\left.\mathbf{2 G}\right)$. Additionally, seal the damaged peripheral airway with a surgical clamp (arrow) (Figure $\mathbf{2 H}$ ).

12. Apply agarose until the lung tissue is filled completely. Do not over-inflate the tissue as this may cause irreversible damage to the tissue structure and its cells.

13. Clamp the bronchus that was used for the filling immediately. Remove the cannula prior to clamping.

14. Incubate the tissue in the culture medium at $4{ }^{\circ} \mathrm{C}$ for $30 \mathrm{~min}$ to ensure agarose solidification.

15. If the resected tissue has multiple bronchial entries, repeat step 4.2 to 4.13 until all parts of tissue are filled with agarose.

16. Store the agarose filled lung tissue sections in $4{ }^{\circ} \mathrm{C}$ cold medium until slicing.

\section{Precision-cut Lung Slicing}

1. Identify regions in the lung tissue that are solidly filled with the agarose. Solidly filled regions will not collapse when they are gently pressed with tweezers against the bottom of the cell culture dish.

1. Excise a $1-1.5 \mathrm{~cm}^{3}$ block of regions described in 5.1 , whereas one side still should be covered with pleura.

2. Attach each individual tissue block with the pleural side contacting the holder of the vibratome by using a cyanoacrylate glue.

NOTE: The pleura is slightly elastic and therefore impedes the cutting with the vibratome blade. Placed on the tissue holder, the pleura will not interfere with the cutting and, importantly, forms a natural barrier between the cyanoacrylate glue and the tissue's parenchyma allowing for minimal diffusion of the glue.

3. Slice the lung tissue with the vibratome with the following settings: thickness: $500 \mu \mathrm{m}$, frequency: $100 \mathrm{~Hz}$, amplitude of the knife: $1.2 \mathrm{~mm}$, forward speed of the blade of 3-12 $\mu \mathrm{m} / \mathrm{s}$, which depends on tissue stiffness. Reduce the protrusion-speed of the blade if the slice is not cut properly, or if the tissue block itself starts to vibrate.

4. Gently transfer the slice by lifting it with forceps from the vibratome tray into a well of a 12-well plate filled with cultivation medium. Finally, incubate the lung slices in an incubator under standard cell culture conditions.

5. Stop slicing when $2-3 \mathrm{~mm}$ of the tissue block are left unsliced since the cyanoacrylate glue may have compromised the tissue integrity of this region.

\section{Generation of PCLS Punches}

1. Transfer the lung slices from a single well to an empty $10 \mathrm{~cm}$ dish.

2. Place a $4 \mathrm{~mm}$ biopsy puncher orthogonally to the upper surface of a PCLS and start to move the puncher in clockwise and counterclockwise rotations.

3. Fill cell culture medium into the wells of a 96-well plate. Lift the tissue punches with forceps and transfer the punches into the wells of a 96-well plate. Finally, incubate the lung punches submerged in the medium prepared in 1.1.1. in a cell culture incubator under standard conditions $\left(21 \%(\mathrm{v} / \mathrm{v})\right.$ oxygen, $5 \%(\mathrm{v} / \mathrm{v})$ carbon dioxide and $95 \%$ humidity, at $\left.37^{\circ} \mathrm{C}\right)$.

\section{Tissue Culture and Sample Harvesting}

1. Culture the PCLS and punches overnight in an incubator under standard cell culture conditions.

2. Culture PCLS and punches under outlined condition for a maximum of 120 hours after their generation to ensure cellular viability and function.

3. For harvesting protein as well as RNA, wash PCLS and punches three times in phosphate buffered saline (PBS), transfer them into cryovials and snap-freeze in liquid nitrogen.

4. Sample medium supernatant of cultured PCLS punches for the analysis of secreted proteins.

1. For histological analyses, wash the PCLS and punches three times with PBS and fix them with $4 \%$ paraformaldehyde by incubating for 30 min at $37^{\circ} \mathrm{C}$. Finally, store the PCLS in PBS at $4{ }^{\circ} \mathrm{C}$ for further downstream staining 


\section{Representative Results}

\section{PCLS generation}

The generation of PCLS can be separated into four essential steps: surgical lung tissue resection, agarose filling, vibratome-based PCLS generation, and culture of PCLS. The resected lung tissue is filled with low-melting point agarose, which adds the required stiffness to the lung tissue for slicing and preserves the native lung structure and architecture. Of note, PCLS generation is highly time consuming, thus often overnight storage of the filled lung tissue in DMEM F-12 medium can be included as an additional step and PCLS generation is started on the next day. Depending on the following experimental setup, generated PCLS can be incubated overnight in standard cell culture medium containing $0.1 \%(\mathrm{w} / \mathrm{v})$ fetal bovine serum, before experimental conditions are applied. 3D-LTCs were viable and exhibited cellular functionality (such as surfactant protein secretion) for up to $120 \mathrm{~h}^{22}$ in the culture conditions outlined in this protocol (Figure 1) and might be optimized upon further improvement thereof.

\section{Agarose filling}

For agarose filling of the tissue, a cannula of a peripheral venous catheter with a $1.3 \mathrm{~mm}$ diameter attached to the agarose-filled syringe was inserted into a bronchus at the surface of the cut tissue (Figure 2A). Bronchi are often localized near to a pulmonary artery. While the arteries have thinner walls and tend to collapse, bronchi exhibited a good visible lumen. Depending on the tissue's integrity, the catheter can be advanced through several generations of the respiratory tree into the periphery of the lung. The penetrated bronchus was sealed around the cannula by using tweezers (Figure 2B). The pulmonary artery can be clamped with the tweezers at the same time. Afterwards, the tissue is lifted up and liquid agarose is gently instilled into the airways.

Depending on the position of the catheter, a majority of the tissue can be filled with liquid agarose (Figure 2D). Optionally, cone like parts of the lung tissue, which reflect the lung's parenchyma ventilated by the penetrated bronchus, might get filled with the agarose (Figure $2 \mathrm{C}$ ). In both scenarios, a characteristic pattern of solidly filled tissue regions can be observed: first, a major part of the tissue is filled in wedges (Figure 2D), or secondly, smaller protruding round areas of thoroughly filled tissue regions appear (Figure 2C). If parts of the airways obstruct due to agarose clots or other causes, parts of the tissue might not be properly filled with agarose. Thus, only parts of the tissue might be applicable for slicing. In case of leakages during the agarose filling procedure, parts of the filled respiratory tree might get perforated and filling of the lung tissue gets nearly impossible However, possible workarounds include the filling via a more peripheral bronchus, a deeper penetration of the cannula into the distal airways (Figure $\mathbf{2 G}$ ), or potential clamping of the leakage area (Figure $\mathbf{2 H}$ ).

\section{Precision-cut lung slicing}

Tissue blocks at a length and width of $1-1.5 \mathrm{~cm}$ were excised from tissue regions, which were completely filled with solidified agarose (Figure 3A-3B). Next, the individual tissue blocks were glued on the tissue holder of the vibratome (Figure 3C). $500 \mu \mathrm{m}$ thick PCLS were generated, whereas the tissue block at the vibratome was advancing forward with speeds between 3-12 $\mu \mathrm{m} / \mathrm{s}$. (Figure 3D-3F). Finally, the PCLS were submerged in cell culture medium containing $0.1 \%(\mathrm{w} / \mathrm{v})$ fetal bovine serum and cultured at standard cell culture conditions, as outlined step 7.

\section{Experimental readouts of human 3D-LTC after $48 \mathrm{~h}$ of culturing}

A representative immunofluorescence staining, as previously described by Alsafadi et al ${ }^{25}$, is shown in Figure $4 \mathrm{~A}-4 \mathrm{C}$. Immunolabeling of fibronectin (red) and cell nuclei (DAPI, blue), allowed for imaging of the preserved alveolar structure in the human 3D-LTC ex vivo. Treatment of the human PCLS punches with a profibrotic cytokine cocktail (including transforming growth factor beta 1, platelet derived growth factor AB, lipophosphatidyl acid, and tumor necrosis factor alpha) for $48 \mathrm{~h}$ resulted in fibrosis-like changes in human 3D-LTCs. By qPCR, a significant induction of the fibrosis-relevant extracellular matrix components collagen type 1 and fibronectin genes in 3D-LTC punches was observed upon treatment with the profibrotic cocktail (Figure 4D). Additionally, protein levels of the mesenchymal marker vimentin were found upregulated in 3 out of 4 patients after treatment of 3D-LTC punches (Figure 4E).

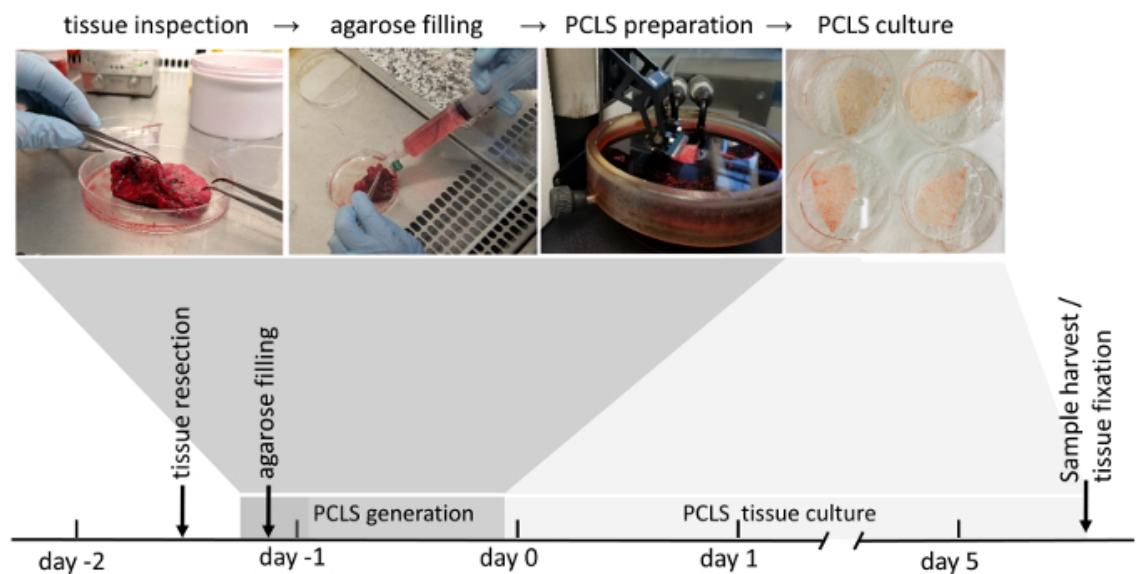

Figure 1: Workflow of PCLS generation. Tumor-free areas of lung resections are thoroughly inspected due to their tissue integrity. If the tissue is scored suitable for further use (scoring is explained in detail in the material and methods section), it is next filled with liquid agarose. Tissue blocks filled with solidified agarose are subsequently sliced with a vibratome. Submerged in cell culture medium, 3D-LTC are cultured up to 120 $\mathrm{h}$ after their generation. Downstream analyses of the 3D-LTCs involve protein- or RNA-expression, live-tissue fluorescence imaging, as well as immunofluorescence staining after fixation of the tissue. Please click here to view a larger version of this figure. 
A

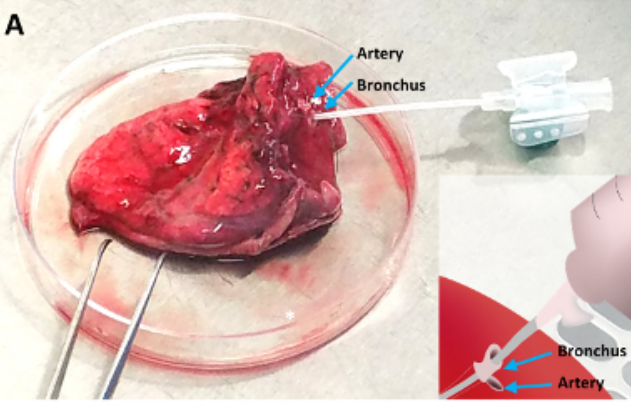

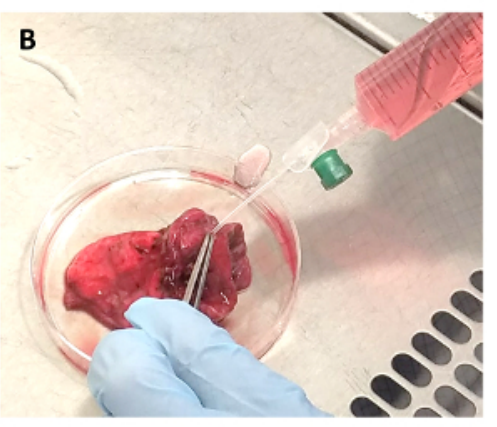

B

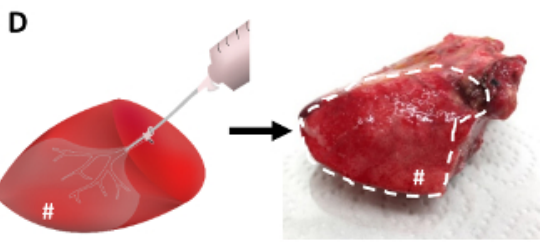

G

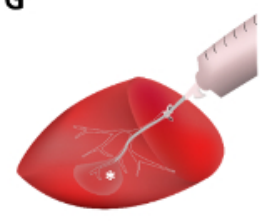

$\mathbf{H}$

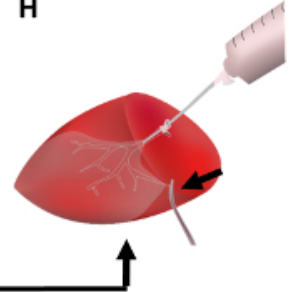

Figure 2: Filling the lung tissue with low-melting point agarose. The lung tissue is cannulated with a peripheral venous catheter which is inserted into a bronchus adjacent to the pulmonary artery (Figure 2A). Tweezers are used to fix the cannula in the bronchus and to clamp the pulmonary artery to avoid leaking of the liquid agarose. Liquid agarose at $42{ }^{\circ} \mathrm{C}$ is poured into the lung tissue with a $30 \mathrm{~mL}$ syringe (Figure $\left.2 \mathrm{~B}\right)$. A distal positioning of the cannula during filling will result in small areas of filled tissue (Figure $2 \mathrm{C}$ ), while proximal positioning will ensure the filling of a larger tissue volume (Figure 2D). Any obstructions of the airways will reduce the amount of tissue volume that can be filled (Figure 2E). In case of agarose leaking, a distal cannula positioning and/or clamping of the leakage side enables proper agarose filling of the lung tissue (Figure 2F-2H). Please click here to view a larger version of this figure.

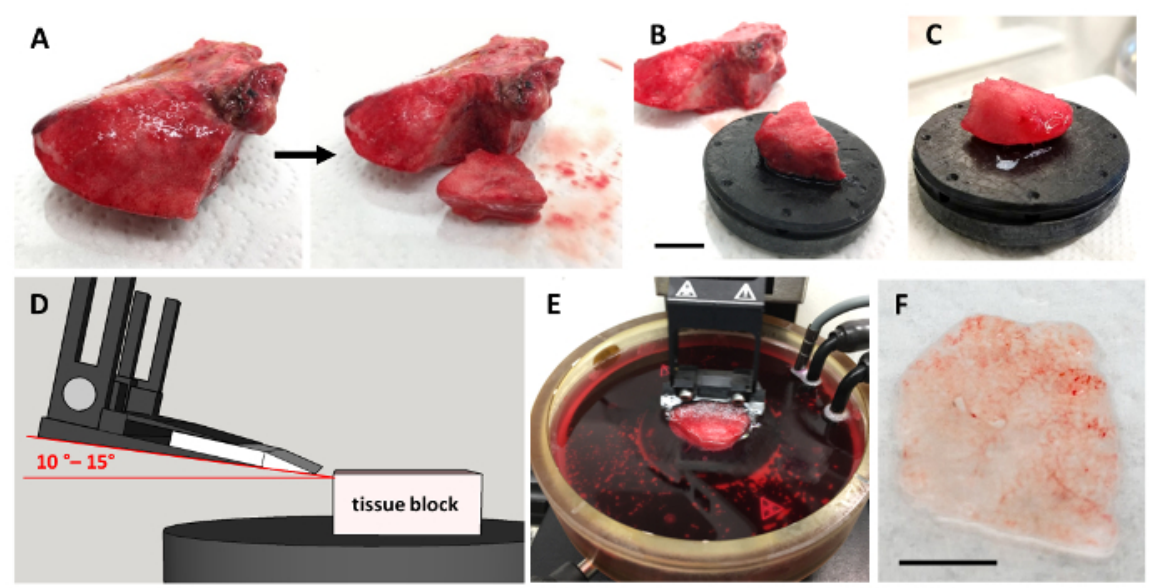

Figure 3: Precision-cut lung slicing. A successfully agarose-filled lung tissue is used to excise a piece of a tissue block (1 cm $x 1.5 \mathrm{~cm} \times 1 \mathrm{~cm}$ ) with a scalpel (Figure 3A). Next the excised tissue block is glued to the tissue holder, scale bar indicates $1 \mathrm{~cm}$ (Figure 3B). Preferably, the tissue is glued with its pleural surface to the surface of the tissue holder as shown in Figure 3C. $500 \mu \mathrm{m}$ thick slices are cut by the vibratome with a sapphire knife in a $10^{\circ}-15^{\circ}$ angle relative to the tissue (Figure 3D and 3E). The cutting procedure results in $2-3 \mathrm{~cm}^{3}$ large intact lung slices, Scale bar $=5 \mathrm{~mm}$ (Figure 3F). Additionally, by using a biopsy puncher, small reproducible punches with a diameter of $4 \mathrm{~mm}$ can be generated. Please click here to view a larger version of this figure. 

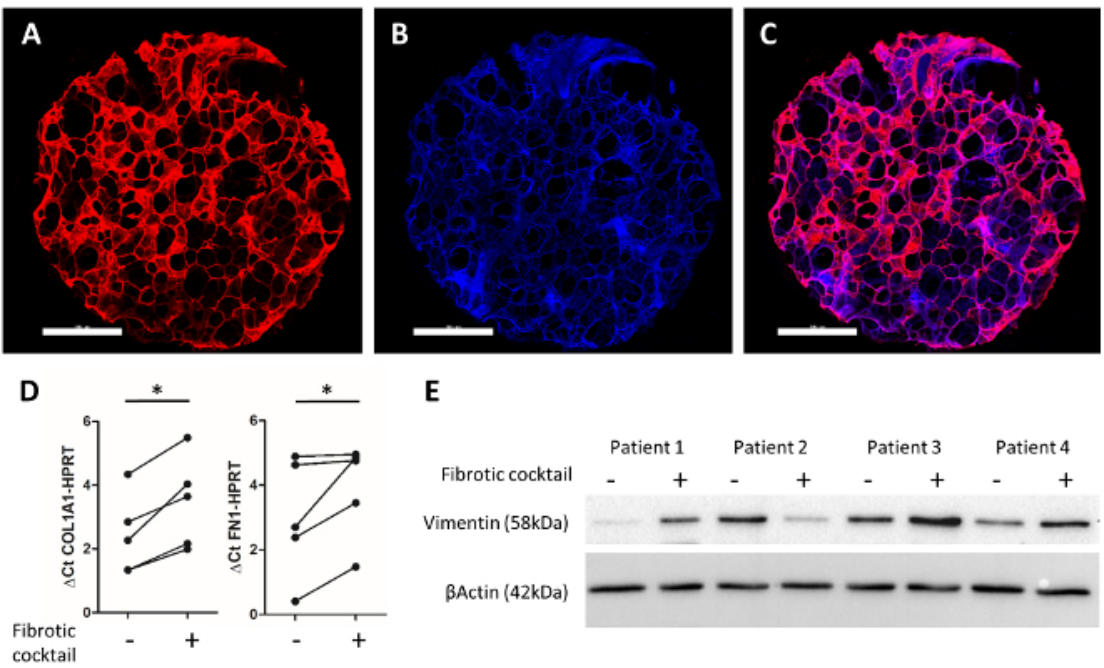

Figure 4: Experimental readouts of human 3D-LTCs after $48 \mathrm{~h}$ of culture. A human 3D-LTC punch of 4 mm diameter was immunostained for fibronectin (in red) and DAPI (in blue) (Figure 4A-4C). Scale bars $=1,000 \mu \mathrm{m}$. Figure 4C shows the merged image. RNA analysis of PCLS by quantitative RT-PCR shows significant increases of COL1A1 and FN1 gene expression by the profibrotic cocktail ${ }^{25}$. Figure $4 \mathrm{E}$ displays an immunoblot of whole protein lysates of PCLS, which were treated with a fibrotic cocktail ${ }^{25}$. Probing for Vimentin and $\beta$-Actin demonstrated an increased protein expression of the mesenchymal marker (vimentin) after treatment with profibrotic factors in patient samples 1,3 , and 4 . Please click here to view a larger version of this figure.

\begin{tabular}{|c|c|}
\hline Criterion & Points \\
\hline The tissue sample has intact pleural surface. & 20 \\
\hline $\begin{array}{l}\text { The tissue sample seems macroscopically intact, lacking incisions, } \\
\text { squashing, ruptures and distortions. }\end{array}$ & 20 \\
\hline $\begin{array}{l}\text { The tissue sample contains at least one bronchus with a diameter } \\
>1 \mathrm{~mm} \text {. }\end{array}$ & 20 \\
\hline The tissue sample contains no or only little amounts of blood. & 4 \\
\hline $\begin{array}{l}\text { The tissue sample was stored fully in medium and shows no obvious } \\
\text { sign of atelectasis. }\end{array}$ & 4 \\
\hline The tissue sample was resected within the last four hours. & 4 \\
\hline The tissue sample is larger than $5 \mathrm{~cm}$ in its largest diameter. & 4 \\
\hline Score in sum: & \\
\hline
\end{tabular}

Table 1: Lung Agarose Filling Score. The Lung Agarose Filling Score (LAFS) correlates with the success-rate to fill a tissue resection with agarose for its subsequent vibratome-based PCLS production. The score sums up all points of criteria met by the tissue. An LAFS equal or above 72 predicts good agarose filling properties, a score below 60 predicts a highly probable failure of agarose filling of the tissue.

\section{Discussion}

The protocol described in this manuscript covers the generation of PCLS from human lung tissue resectates by filling it with liquid agarose and subsequent vibratome slicing. Generation of tissue slices was demonstrated before for a couple of organs, like liver and brain, whereas the inherent stiffness of these organs allowed direct slicing without any modification of the tissue. Of note, the initial proper preparation of the lung tissue is the most crucial step in generating PCLS. Agarose filling of the lung is the method of choice to stabilize its soft and elastic nature, and to ensure a homogenous and reproducible PCLS generation. Large airways of the resected lung tissue are cannulated to provide access to the small airways, as well as to the intact lung parenchyma. The lack of an intact pleura, which makes agarose filling nearly impossible, is a major reason why lung tissue is mostly not usable for lung slicing. Prospectively, a synthetic pleura originally designed to perform functional experiments on decellularized scaffolds could potentially be applied to achieve successful agarose filling of explants that lack an intact pleura ${ }^{31}$ Resections resulting in a human lung tissue piece with intact pleura are essential for generating tissue blocks for slicing. Resected tissue is more available due to tumor-free tissue from cancer resections than fully intact lobes or whole-lung explants of patients who underwent lung transplantation.

Commonly, two systems are used to produce PCLS: the Krumdieck tissue slicer ${ }^{15}$ and vibratory microtomes (vibratomes). Tissue slicers generate slices by passing a tissue block through a metal vessel, which cuts the PCLS at $90^{\circ}$ at the end of this vessel. Vibratomes generate PCLS by moving a vibrating knife horizontally over an anchored block of tissue that is submerged in a cooled medium bath, which compared to the Krumdieck slicer exerts less shear force on the tissue. This results in less harsh treatment of the tissue before cultivation. On the other hand, the vibratome cutting is more time and work consuming. In our hands, vibratome slicing enabled the production of a maximum of 100 PCLS 
or 500 PCLS punches in one day, sufficient for most experimental studies. PCLS can be cultured in various ways: (a) attached to Trans-wells, thus generating an air liquid interface (ALI) system, (b) as dynamic organ culture (DOC), or (c) submerged in cell culture medium at standard cell culture conditions. The in-detail cultivation of PCLS was previously described ${ }^{22,23,25}$; however, a common standard of cultivation conditions between their usage in various labs around the world is still missing. In particular, the culture time might be critical: as in murine PCLS, a loss of SFTPC positive alveolar type 2 cells is observed after $144 \mathrm{~h}$, but not after $120 \mathrm{~h}^{22}$. In addition, metabolic activity seems to remain stable in murine $\mathrm{e}^{22}$ and human $\mathrm{PCLS}^{25}$ for $120 \mathrm{~h}$.

There are a couple of technical limitations for the generation of PCLS: the number and size of the resectates fluctuates over time; the efficiency of the agarose filling, which depends on the presence of intact pleura within the obtained tissue, determines the final success of the PCLS generation; and tissue destruction caused by pathological changes within the obtained (diseased) lung tissue might interfere with the PCLS preparation. Airway obstructions and fibrotic tissue lacking intact alveolar space impede with agarose filling and thus make slicing of the fibrotic tissue a demanding task. Emphysematous tissues as found in diseases such as COPD or alpha-1-anti-trypsin deficiency might not withstand the pressure of agarose filling, and will result in rupture of the alveoli and architectural artefacts. In these cases, the usage of low agarose concentration, e.g., $1 \%(\mathrm{w} / \mathrm{v})$, might be useful to decrease pressure and speed during agarose filling. Overall, the disease state of the tissue can dramatically limit the use of the tissue for PCLS generation. All these parameters determine the amount of PCLS that can be generated from lung tissue, and also the amount of time it takes to produce the PCLS. Further limitations of PCLS are inconsistencies in between different lung slices with respect to size or tissue content, which requires further normalization steps for experiments. To overcome this, biopsy punches of similar regions of the same slice can be generated. This procedure is apt to reduce the tissue variability and, as an additional benefit, increase the number of PCLS samples that can be used for experiments.

In conclusion, human 3D lung tissue cultures from agarose filled PCLS provide a complex human model for studying lung physiology and diseases. The protocol provides a detailed description of the preparation of PCLS from resected lung tissue and their cultivation, and moreover addresses challenges in agarose filling of human lung resections and how to overcome them.

Disclosures

All authors declare no competing financial interests.

\section{Acknowledgments}

The authors are grateful to Marisa Neumann for expert technical assistance. All lung tissues were kindly provided by the CPC-M Bio-Archive. This work was supported by the German Center of Lung Research (DZL), the Helmholtz Association and CPC Research School grants.

\section{References}

1. Lozano, R. et al. Global and regional mortality from 235 causes of death for 20 age groups in 1990 and 2010: a systematic analysis for the Global Burden of Disease Study 2010. Lancet. 380 (9859), 2095-2128, (2012).

2. Rosenberg, S. R., Kalhan, R., \& Mannino, D. M. Epidemiology of Chronic Obstructive Pulmonary Disease: Prevalence, Morbidity, Mortality, and Risk Factors. Seminars in Respiratory and Critical Care Medicine Med. 36 (4), 457-469, (2015).

3. Hekking, P. P. et al. The prevalence of severe refractory asthma. The Journal of Allergy and Clinical Immunology. 135 (4), 896-902, (2015)

4. Woodard, G. A., Jones, K. D., \& Jablons, D. M. Lung Cancer Staging and Prognosis. Cancer Treatment and Research. 170 47-75, (2016).

5. Ley, B., Collard, H. R., \& King, T. E., Jr. Clinical course and prediction of survival in idiopathic pulmonary fibrosis. American Journal of Respiratory and Critical Care Medicine. 183 (4), 431-440, (2011).

6. Burgstaller, G. et al. The instructive extracellular matrix of the lung: basic composition and alterations in chronic lung disease. European Respiratory Journal. 50 (1), (2017).

7. Degryse, A. L., \& Lawson, W. E. Progress toward improving animal models for idiopathic pulmonary fibrosis. The American Journal of the Medical Sciences. 341 (6), 444-449, (2011).

8. Fricker, M., Deane, A., \& Hansbro, P. M. Animal models of chronic obstructive pulmonary disease. Expert Opinion on Drug Discovery. 9 (6), 629-645, (2014).

9. Sagar, S., Akbarshahi, H., \& Uller, L. Translational value of animal models of asthma: Challenges and promises. European Journal of Pharmacology. 759 272-277, (2015).

10. Williamson, J. D., Sadofsky, L. R., \& Hart, S. P. The pathogenesis of bleomycin-induced lung injury in animals and its applicability to human idiopathic pulmonary fibrosis. Experimental Lung Research. 41 (2), 57-73, (2015).

11. Cooper, P. R. et al. Formoterol and salmeterol induce a similar degree of beta2-adrenoceptor tolerance in human small airways but via different mechanisms. British Journal of Pharmacology. 163 (3), 521-532, (2011).

12. Skronska-Wasek, W. et al. Reduced Frizzled Receptor 4 Expression Prevents WNT/beta-Catenin-driven Alveolar Lung Repair in Chronic Obstructive Pulmonary Disease. American Journal of Respiratory and Critical Care Medicine. 196 (2), 172-185, (2017).

13. Lehmann, M. et al. Senolytic drugs target alveolar epithelial cell function and attenuate experimental lung fibrosis ex vivo. European Respiratory Journal. 50 (2), (2017).

14. Koch, A. et al. Murine precision-cut liver slices (PCLS): a new tool for studying tumor microenvironments and cell signaling ex vivo. Cell Communication and Signaling. 12 73, (2014).

15. Ebsen, M. et al. Infection of murine precision cut lung slices (PCLS) with respiratory syncytial virus (RSV) and chlamydophila pneumoniae using the Krumdieck technique. Pathology - Research and Practice. 198 (11), 747-753, (2002).

16. Kennedy, J. L. et al. Effects of rhinovirus 39 infection on airway hyperresponsiveness to carbachol in human airways precision cut lung slices. The Journal of Allergy and Clinical Immunology. 141 (5), 1887-1890 e1881, (2018).

17. Royce, S. G. et al. Airway Remodeling and Hyperreactivity in a Model of Bronchopulmonary Dysplasia and Their Modulation by IL-1 Receptor Antagonist. American Journal of Respiratory Cell and Molecular Biology. 55 (6), 858-868, (2016). 
18. Donovan, C. et al. Rosiglitazone elicits in vitro relaxation in airways and precision cut lung slices from a mouse model of chronic allergic airways disease. American Journal of Physiology-Lung Cellular and Molecular Physiology. 309 (10), L1219-1228, (2015).

19. Zscheppang, K. et al. Human Pulmonary 3D Models For Translational Research. Biotechnology Journal. 13 (1), (2018).

20. Fisher, R. L. et al. The use of human lung slices in toxicology. Human \& Experimental Toxicology. 13 (7), 466-471, (1994).

21. Wang, L. et al. Differences between Mice and Humans in Regulation and the Molecular Network of Collagen, Type III, Alpha-1 at the Gene Expression Level: Obstacles that Translational Research Must Overcome. International Journal of Molecular Sciences. 16 (7), 15031-15056, (2015).

22. Uhl, F. E. et al. Preclinical validation and imaging of Wnt-induced repair in human 3D lung tissue cultures. European Respiratory Journal. 46 (4), 1150-1166, (2015).

23. Switalla, S. et al. Natural innate cytokine response to immunomodulators and adjuvants in human precision-cut lung slices. Toxicology and Applied Pharmacology. 246 (3), 107-115, (2010).

24. Banerjee, A. et al. Trichostatin A abrogates airway constriction, but not inflammation, in murine and human asthma models. American Journal of Respiratory Cell and Molecular Biology. 46 (2), 132-138, (2012).

25. Alsafadi, H. N. et al. An ex vivo model to induce early fibrosis-like changes in human precision-cut lung slices. American Journal of Physiology-Lung Cellular and Molecular Physiology. 312 (6), L896-L902, (2017).

26. Westra, I. M., Oosterhuis, D., Groothuis, G. M., \& Olinga, P. Precision-cut liver slices as a model for the early onset of liver fibrosis to test antifibrotic drugs. Toxicology and Applied Pharmacology. 274 (2), 328-338, (2014).

27. Vatakuti, S., Schoonen, W. G., Elferink, M. L., Groothuis, G. M., \& Olinga, P. Acute toxicity of CCl4 but not of paracetamol induces a transcriptomic signature of fibrosis in precision-cut liver slices. Toxicology in Vitro. 29 (5), 1012-1020, (2015).

28. Poosti, F. et al. Precision-cut kidney slices (PCKS) to study development of renal fibrosis and efficacy of drug targeting ex vivo. Disease Models \& Mechanisms. 8 (10), 1227-1236, (2015).

29. Li, M., de Graaf, I. A., \& Groothuis, G. M. Precision-cut intestinal slices: alternative model for drug transport, metabolism, and toxicology research. Expert Opinion on Drug Metabolism \& Toxicology. 12 (2), 175-190, (2016).

30. Morin, J. P. et al. Precision cut lung slices as an efficient tool for in vitro lung physio-pharmacotoxicology studies. Xenobiotica. 43 (1), 63-72, (2013).

31. Wagner, D. E. et al. Design and Synthesis of an Artificial Pulmonary Pleura for High Throughput Studies in Acellular Human Lungs. Cellular and Molecular Bioengineering. 7 (2), 184-195, (2014). 